\title{
Begeisterung für den schönsten Beruf der Welt
}

\author{
Ärztemangel in ländlichen Regionen wird seit vielen Jahren beklagt. Im Bayerischen Wald gehen \\ vier Kollegen das Problem aktiv an. Ihr Projekt LandArztMacher lädt Dutzende von Medizinstuden- \\ ten für Praktika und Schnuppertage ins Arberland - mit messbarem Erfolg.
}

_ Mit Stipendien, finanziellen Anreizen und anderen Erleichterungen versucht man vielerorts, junge Mediziner aufs Land zu locken - mit wechselndem Erfolg. Vier Ärzte aus dem Bayrischen Wald gehen einen anderen Weg. Für sie, die sich als Macher verstehen, ist der Landarzt der Traumberuf schlechthin. Genau das wollen sie angehenden Ärzten vermitteln: durch Praktika, Ausbildungsmodelle, persönliche Betreuung und Vernetzung in der Region. Für ihr Engagement sind die „LandArztMacher" mit dem 3. Preis des von Springer Medizin vergebenen CharityAwards ausgezeichnet worden.

Als „Investition in die Zukunft“ betrachtet PD Martin Kammerl, Facharzt für Innere Medizin, Nephrologie und Dialyse mit einer Praxis am Zwieseler Kreiskrankenhaus, das Programm, das er 2013 gemeinsam mit seinen Kollegen Dr. Jana Riedl, Intenistin am Kreiskrankenhaus Viechtach, Hausarzt Dr. Wolfgang Blank aus Kirchberg, sowie Dr. Martin Müller, Orthopäde und Unfallchirurg am Kreiskrankenhaus Zwiesel, entwickelt hat. „Es ist wichtig, die Medizinstudenten auf ihrem Weg in die Ausbildung zu unterstützen“, so Kammerl. „Und das ist manchmal mit Aufwand verbunden.“

\section{Garantiert hervorragende ärztliche Weiterbildung}

Zwiesel, Viechtach und Kirchberg liegen im Arberland im Landkreis Regen, in dessen 24 Gemeinden etwa 78.000 Einwohner leben. Hier haben die „LandArztMacher“ vor drei Jahren ihre „Exzellent“-Initiative gestartet, in der sie angehenden Medizinern eine „hervorragende ärztliche Weiterbildung“ garantieren. Während ihrer Praktika lernen die Jungärzte sowohl den Alltag eines Landarztes als auch das „Land“ selbst kennen und schätzen - oder manchmal sogar lieben. „Wir vermitteln ihnen positive Modelle für die Vereinbarkeit von Fa-

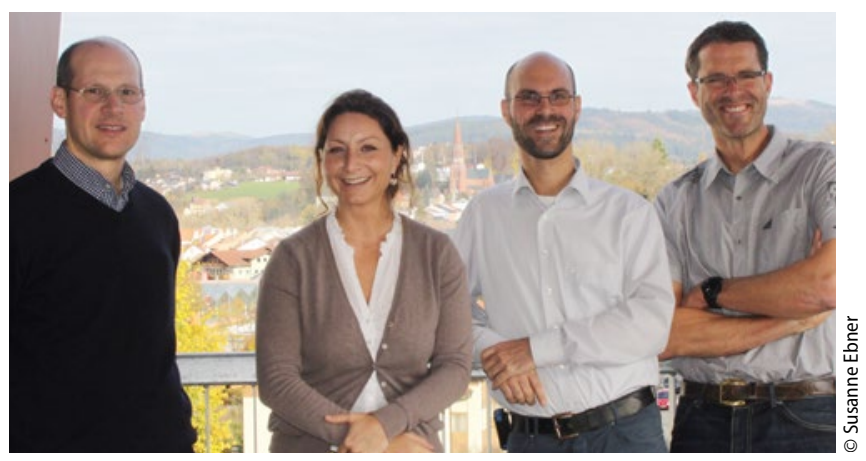

Die LandArztMacher (v. I.): Dr. Martin Kammerl, Dr. Jana Riedl, Dr. Martin Müller und Dr. Wolfgang Blank.

\section{CharityAward 2016}

Mit dem CharityAward zeichnet Springer Medizin seit 2011 das herausragende Engagement von Stiftungen, Organisationen und Institutionen aus, die sich in besonderer Weise der Gesundheitsversorgung in Deutschland verpflichtet fühlen. Schirmherr ist der Bundesminister für Gesundheit Hermann Gröhe. Dotiert ist die Auszeichnung mit einem Preisgeld von 60.000 Euro, aufgeteilt auf insgesamt drei Gewinner. Auch 2016 wird der Preis wieder vergeben, Interessierte können sich bis zum 30. Juni online bewerben: www.springermedizin.de/charity

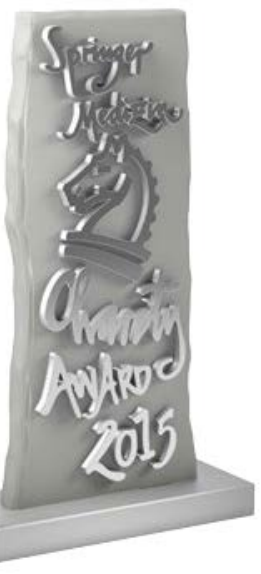

milie und Beruf sowie eine ausgewogene Work-Life-Balance“, erklärt Hausarzt Blank. Wichtig sei auch die Erfahrung aktiv gelebter Netzstrukturen.

Jeweils im März und August laden die „LandArztMacher“ bis zu 23 Studenten zu vierwöchigen Gruppenpraktika in die Region ein. Hinzu kommen jedes Jahr vier Thementage mit 20 Medizinstudenten und ein Themenwochenende mit mehr als 50 Studenten. Während ihres Aufenthaltes in der Region werden die angehenden Ärzte intensiv von erfahrenen Kollegen betreut, die ihnen auf Augenhöhe begegnen. Fortbildungen etwa in Sonografie, Blutentnahme und EKG ergänzen die praktische Anschauung. Dank einer breiten Unterstützung durch den Landkreis, ortansässige Firmen und Krankenkassen haben die Studenten während ihres Aufenthalts freie Kost und Logis. Ausflüge schaffen eine Bindung zur Region. „Wir wollen den jungen Ärzten zeigen, dass der Bayerische Wald eine gute Existenzgrundlage sein kann“, sagt Blank.

Einer eigenen Evaluation zufolge haben er und seine Kollegen mit ihrer Initiative tatsächlich Erfolg. Nach der Teilnahme verbessert sich bei den Jungmedizinern sowohl die Haltung zum Arztberuf als auch die Einstellung zum Landleben. „Wir meinen: Landarzt zu sein, ist der schönste Beruf“, erklärte Blank bei der Verleihung der CharityAwards in Berlin.

Pete Smith

VIDEO-INTERVIEWS AUF SPRINGERMEDIZIN.DE

Dr. Wolfgang Blank spricht über die LandÄrzteMacher

$\rightarrow$ www.springermedizin.de/6197966 